\title{
Spatial-Temporal Simulation to Estimate the Load Demand of Battery Electric Vehicles Charging in Small Residential Areas
}

\author{
Joel D. Melo • Edgar M. Carreno • \\ Antonio Padilha-Feltrin
}

Received: 22 July 2013 / Revised: 19 December 2013 / Accepted: 24 January 2014 / Published online: 19 February 2014

(C) Brazilian Society for Automatics-SBA 2014

\begin{abstract}
This paper presents a spatial-temporal approach for estimating the load demand of battery electric vehicles (BEV) charging in small residential areas. This approach is especially suited for simulating the driving pattern of BEVs in cities without this kind of information. The service zone is divided into several sub-zones; each of these has a probability that represents how likely it is for a BEVs to cross the sub-zone. The driving pattern of BEVs is simulated using a multi-agent framework, which estimates the spatial distribution of these in a city. To determine the hourly charge in each place identified in the spatial area, the model considers the battery charging profile via two charging scenarios. The main contribution of this method is the estimation of $\mathrm{BEV}$ charging in feeders or transformers using small-scale simulation. The proposed approach was tested on a real distribution system of a mid-sized city in Brazil. For this specific system, the simulation was able to identify two different levels of agglomerations; when the worst-case scenario with a $20 \%$ penetration is analyzed, an increase in peak demand up to $34.04 \%$ was determined in the most affected part of the distribution system while the rest of the distribution system is almost unaffected.
\end{abstract}

Keywords Battery electric vehicle - Distribution planning Load estimation · Multi-agent · Percolation theory.

J. D. Melo $(\bowtie) \cdot$ A. Padilha-Feltrin

Department of Electrical Engineering, University of the State

of Sao Paulo-UNESP, Ilha Solteira, SP, Brazil

e-mail: joeldavidm@gmail.com

E. M. Carreno

Center for Engineering and Mathematical Sciences-CECE, University of the State University of West Parana-UNIOESTE, Iguaçu, PR, Brazil

\section{Variables}

$v$

$p_{c}$

$p$

$E_{i}$

$S O C_{i}$

$d$

$d_{R}$

$t$

$k$

$D$

$S$

$S\left(p_{c}\right)$

$D_{j}$

$\mathrm{F}(\mathrm{k})$

$\operatorname{prob}\left(S_{j-(t-k)}\right)$

$\Phi\left(D_{j}, t\right)$

$n$

$n_{c}$

$\mathrm{P}_{\mathrm{n}}$
Connectedness length.

Connectedness length exponent.

Minimum state of charge required for the BEV to cross the entire city.

Random variable associated to state of charge of the battery.

Residual state of charge of BEV battery $i$.

State of charge used in the beginning of the simulation of driving patterns for a BEV battery i.

Daily distance traveled by BEV.

Maximum range of the BEV at the time that the SOC meter indicates "empty."

Time instant $(1 \leq t \leq 24)$.

Time instant $(k \leq t, 1 \leq k \leq 24)$.

Power level.

Initial charge of a BEV battery.

Charge associated to $P_{C}$ in the simulation of driving patterns.

Power level at half-hourly interval $\mathrm{j}$.

Probability of charge starting at time instant $k$.

Probability of an initial charge being at power level $S_{j-(t-k)}$.

Probability of a battery charge starting at time instant $K$ and charging load operating at power level $D_{j}$ at time instant $t$.

Total number of BEVs in each sub-area. Total number of half-hourly intervals.

Total power demand for BEV battery chargers. 
$c$

$\mathrm{F}_{\mathrm{A}}(\mathrm{t})$

$F_{B}(t)$
Random number used at the beginning of the multi-agent simulation. Discrete distribution used in first charging scenario.

Discrete distribution used in second charging scenario.

\section{Introduction}

Plug-in electrical vehicles are classified depending on how they receive electrical energy, it could receive energy only from the grid battery electric vehicles (BEVs), from an internal combustion engine hybrid electric vehicle (HEV) or a combination of both methods plug-in hybrid electric vehicle (PHEV).(Tuttle and Baldick 2012) This paper deals with the spatial-temporal charging of BEVs in small scale.

From a distribution planning perspective, BEVs are unknown quantities, representing a potential demand which varies both spatially and temporally in the distribution network. While the overall electric system capacity might be sufficient, there could be local network problems related to network capacity due to an agglomeration of BEVs. Because of these characteristics, the electric distribution utilities must consider spatial-temporal studies to improve the modeling for the charging loads of BEVs.

Generally, the load of BEVs is expressed as the penetration or agglomeration of BEV chargers in a distribution system. This penetration is the ratio of the overall number of BEV chargers to the total number of residences . (Staats et al. 1997) Because there are no actual data for concentration of $\mathrm{BEV}$ chargers, modeling the BEV load is more difficult than modeling the demand load in a distribution system. However, a concentration of BEV chargers can be predicted if the randomness of individual charger start-time and initial battery state-of-charge (SOC) are properly considered (Staats et al. 1997).

Several studies (Qian et al. 2011; Deilami et al. 2011) have already been carried out to calculate the overall effect on load demand of the BEVs, considering a global penetration of BEVs for simulations in the service area; however, none of these techniques are capable of simulating the charging of BEVs in a spatial form, considering small-area resolution; thus they cannot facilitate the identification of small areas with a high penetration of BEVs. Furthermore, although the traffic-habit information is considered as given input, this information is generally extracted from large cities, specially in the US, that do not necessarily reflects drivers behaviors in small cities around the world.

Currently, some cities in the world have an urban-planning area for the public transport systems, where a traffic flow simulation is performed (Batty 2007). In this case, these simulations determine the driving patterns of BEVs. However,

this planning is not done in cities in developing countries. Thus, the electrical distribution utilities for such cities do not have information about driving patterns. This paper presents a methodology to estimate the charge of BEVs in cities without this kind of information. This methodology uses smallarea resolution and a simulation to establish the spatial distribution of these devices by mimicking the driving pattern of BEVs, and using the battery charging profiles to model the hourly recharge in each place that was identified in the spatial distribution.

The first step in the process of modeling the charging of BEVs is to determine the residual SOC. This SOC could be calculated using the distance that a BEV traveled. In this work, it is considered that electrical distribution utilities do not have information about this distance, and so, a multiagent framework is used to simulate it.

In summary, this paper presents a spatial-temporal model to determine the charging of BEVs, modeling their hourly load demand in small areas. Planners can use the output of the model to know which feeders/substations will be affected by BEVs depending on their penetration level. This model could be used using information easily available in the most distribution utilities.

\section{Modeling the Charging of BEVs}

Traditional forecasting techniques are not useful for modeling the load demand from BEVs in a distribution system because of the lack of any historical data and considerable uncertainty regarding the future of BEVs. The load of BEVs depends on the future course followed by many factors, such as: (i) market conditions and public acceptance, (ii) battery charging profile, and (iii) availability of charging facilities and other factors, as explained by Rahman and Shrestha (1993). In order to generate historical data for BEVs, some live labs were created around the world, like small cities that would be models for automobile-dependent development patterns (Roe et al. 2008; Évora - InovCity 2010; KEMA and ISO / RTO Council 2010).

Modeling the charging pattern of BEVs helps to determine the increase of electrical demand, which could show significant overloads on certain parts of distribution systems, even though the distribution system as a whole may have sufficient or excess capacity at any instant of time (Rahman and Shrestha 1993). Several studies have been conducted inside electrical distribution utilities that used various techniques taking into account the different charging scenarios for BEVs, as shown by Meliopoulos et al. (2009) and Deilami et al. (2011).

The first techniques used the charging load profile from a charging cycle (Rahman and Shrestha 1993), in which different factors are considered to obtain the impact of PEV 
demand on the electric utility distribution network. This practice was later improved using advanced battery systems for PEVs (Valsera-Naranjo et al. 2009) . Various charging proposals have been studied for this problem, such as charging station deployment (Etezadi-Amoli et al. 2010), stochastic load-demand formulation of chargers in distribution systems (Qian et al. 2011), and more recently, optimal charging profile and coordination (Clement-Nyns et al. 2010). All these studies determine the load curve caused by PEV battery charging for the entire service, considering the database of US- driving patterns as given input. However, this database could not easily be used for some electric distribution utilities. Furthermore, none of these methodologies are capable of simulating the area spatially and, in addition, identifying the higher level of local agglomeration of BEVs in distribution system.

Melo et al. (2012) explained that a multi-agent system with a percolation approach could estimate the driving pattern in a scenario of uncontrolled charging load of PEVs considering a flat charging profile. The drawback of this approach is that it cannot show the increase in daily peak demand on feeders or transformers.

The proposed model presents an improvement over these previous methods by taking into account the small-scale simulation to estimate charging of BEVs in feeders or transformers. This small-scale resolution is considered to improve the simulation in small areas with an agglomeration of BEVs.

\section{Spatial-Temporal Models}

Spatial-temporal models are currently used by researchers in urban planning and other similar research fields. With these models, it is possible to simulate the dynamic behavior of different situations in urban centers (for example, traffic on highways or behavior of drivers) and to perform small-scale simulations using simple rules that are easy to implement on a computer platform (Batty 2007). In the planning of road networks studies, the main advantage of these models is to provide information to urban planners for creating new speed limit laws and for determining location and time duration of traffic lights and local traffic jams, among others (Heppenstall et al. 2012) . Because of these characteristics, these models could improve the modeling for charging of BEV loads, as will be shown in this paper. One of the drawbacks of these models is that they need adequate calibration parameters to perform the simulation of dynamic behavior. However, these parameters can be calibrated using the knowledge of experts in the planning area, as explained by Batty (2007).

To model the BEV load demand, the service zone is divided into several sub-zones, and the displacement of BEVs in each sub-zone is characterized with a probability called "network probability", which depends on the main and secondary roads.

\subsection{Spatial Distribution of BEVs}

A multi-agent framework could be used to mimic the behavior of BEV drivers on any system by adjusting the parameters of the simulation, as explained by Batty (2007). Melo et al. (2012) presented a framework composed of a percolation approach in a multi-agent simulation. A percolation model allows calculating the location and distance traveled; so, using this information is possible to calculate the number of sub-zones in the city where each vehicle will move for the random SOC of the battery. Mobile agents are used to represent a set of batteries from BEVs; these agents do not follow a predefined route, instead, they choose their path according to probabilities of each subzone (network probability). The output data of estimation of the spatial distribution of BEVs are the final SOC of batteries after the displacement and the distance traveled (Euclidean distance between the departure and the arrival of BEVs).

\subsubsection{Percolation Cluster Approach}

The origin of percolation theory is related to a publication by Broadbent and Hammersley (1957).

The concepts on how the percolation theory can be used to determine the distance traveled for each PEV through the connectedness length was introduced by Melo et al. (2012):

$\xi \sim\left|p-p_{c}\right|^{-v}$

where $V$ is the connectedness length exponent. The value of $V$ is not universal, but changes continuously with the degree of correlation given by the system. The value of $V$ corresponding to the uncorrelated percolation problem is equal to 1.33, as explained by Makse et al. (1998), for cities characterized by urban growth from the center to the periphery. This work defines the cluster formed as the path taken by BEVs on any common day. Furthermore, the length of these paths is shorter than the distance from the center to the periphery (i.e., short-distance trips).

With the known connectedness length, it is possible to determine the number of sub-zones that can form the path taken by BEVs on any common day considering that the value of $p_{c}$ can be associated to the minimum SOC required for the BEV to travel a distance greater than the distance from the center to the periphery. And the random variable $p$ can be used to characterize the SOC of the battery. Therefore, using Eq. (1) it is possible to calculate the distance traveled by each BEV battery for each random value $p$ (SOC). This work assumes the distance traveled has an inverse power relation between the length of travel and the SOC of each BEV battery, as explained by Heppenstall et al. (2012). 


\subsubsection{Multi-Agent Simulation}

Multi-agent simulations have been used to study the behavior of the different situations in a transport system (Pritchett et al. 2002; Khalesian and Delavar 2008). Batty (2007) and Heppenstall et al. (2012) explain how the agent-based models can simulate the driving patterns. Some considerations or restrictions could be imposed on the agent-based model to get information that can help planners of road networks. For example, a simulation of traffic interactions may consider a slow-speed metric to maintain speed limits on the roads without creating a traffic bottleneck; in this case each agent has a speed controller.

The battery load of the BEV is given to a mobile agent that can travel the city freely through a local movement approach (Carreno and Padilha-Feltrin 2009) until it reaches the connectedness length, calculated using Eq. (1), and stops. Through this equation, each agent places a short trip distance.

The concept of effective travel is considered in the performed simulation of driving patterns, which is calculated as the Euclidean distance between the origin and destination of a trip divided by its real duration. As explained by Heppenstall et al., (2012) this effective travel can be seen as an average speed. The non-linear trend that exists between the average speed and distance traveled may be canceled by reinforcing speed limits or even choosing a location and time duration of traffic lights in the road network. In this work, the shortpaths for BEVs given by the percolation model contribute to the cancelation of this non-linear trend, resulting in their average speed being constant. If the planners need to include traffic jams in the simulation of driving patterns, they should consider the average speed for each BEV (Heppenstall et al. 2012).

The paths of the different agents depend on the characteristics of the sub-zone; each sub-zone holds only information about its own probability of communication to mobile agents and with other neighbor sub-zones. The behavior of $\mathrm{BEV}$ drivers is represented through the interaction between the mobile agent and each sub-zone. The BEV global penetration level determines the total number of electric vehicles in the simulation of driving patterns.

The spatial distribution of BEVs is used to calculate the local agglomeration level of BEVs in each sub-zone. This local agglomeration is used to determine the overall number of BEV batteries that need to be charged in each sub-zone of the city.

The residual SOC of batteries after displacement can be determined using (2), considering that the charge of a BEV drops linearly with the distance traveled (Qian et al. 2011):

$E_{i}=\left(\operatorname{SOC}_{i}-\frac{d}{d_{R}}\right) \times 100 \%$ where $E_{i}$ represents the residual SOC of a BEV battery; $S O C_{i}$ is the SOC used in the beginning of the simulation of driving patterns of a BEV battery $i, d$ is the daily distance traveled by the $\mathrm{BEV}$, which is an output from the multi-agent simulation; and $d_{R}$ is the maximum range of the BEV. In an ideal case, this maximum range would be reached at the time that the SOC meter indicates "empty." A typical value for this is 128.75 km (Mendoza and Argueta 2000; Madrid et al. 1999). Different than Qian et al. (2011), this work considers that the SOC in the beginning of the multi-agent simulation could be different from $100 \%$. This takes into account that residents only recharge their battery when they do not have enough SOC to travel in the city; this could be twice or three times a week and not daily, and only in their residences. Also, the driving pattern is not using average-daily-distance-traveled values for driving; this distance is simulated in the multiagent simulation. This is calculated as the Euclidean distance between the departure and arrival of BEVs, considering that their average speed is constant, which is possible for shortpaths. If the planner wishes to consider traffic jams, then it should consider that the daily distance traveled by the BEV is a non-linear function of average speed, and this can be calculated using the curves presented by Heppenstall et al. (2012).

\subsubsection{Network Probability}

The multi-agent simulation considers that each sub-zone has a probability that represents how likely it is for a mobile agent to cross the sub-zone. This probability depends on the characteristics of the sub-zone and its neighbors, considering the main and secondary roads.

For this work, those probabilities are calculated by binary regression using a generalized additive model (Bivand et al. 2008) in order to get at least the average number of vehicles at crossings.

The generalized additive model was chosen because it allows the use of covariates and a spatial function to perform a preference mapping of the BEV owners to move to a location. The distances to places of interest, such as hospitals, schools, the central business district, and shopping were considered as covariates. The spatial function characterizes the spatial pattern of the BEV owners considering they always have a daily routine to travel to their places of interest. This function can be estimated by considering each road as a set of spatial points. A subroutine in the $R$ software to perform this binary regression is shown by Bivand et al. (2008).

Any other methodology could be used to determine the network probability, like spatial analysis and statistical models, or these probabilities could even be established manually by expert planners using their knowledge of the area and geographic information system (GIS). These methodologies are out of the scope of this paper. These probabilities are con- 
sidered as input data for this work. Further information on the calculation of these probabilities can be found in works published by Heppenstall et al. (2012) and Batty (2007).

\subsection{Hourly Recharge}

The process to determine the hourly recharge is similar to the one explained by Qian et al. (2011) for modeling of load demand due to EV battery charging. The output data from the hourly recharge is the load demand from the BEV batteries charging in each small residential area.

The modeling presented by Qian et al. (2011) considers a probability density functions to determine the likelihood of a battery charge starting at time instant $k \quad(1 \leq k \leq 24)$ and operating at power level $D$ at time instant $k$. The first step in this modeling determines the probability density function for the initial battery charge, considering the probability density function of the distance traveled, this probability is calculated by means of (3) which is derived from what was calculated in the percolation model.

$\operatorname{prob}(S)=\left|S-S\left(p_{c}\right)\right|^{-v}$

where $S$ represents the residual charge of a BEV battery, calculated using Eq. (2); $S\left(p_{c}\right)$ is the charge associated to $p_{c}$ in the simulation of driving patterns in the multi-agent simulation; and $v$ is the connectedness length exponent, as explained in the Sect. 3.1. From this charge, the demand $D$ can be determined during the charging process using the battery charging profile.

Likewise, the demand $D$ during the battery recharge process is discretized with values $D_{j}$ taken at half-hourly intervals to facilitate numerical calculations, as was explained by Qian et al. (2011) .

Figure 1 shows the schematic of an individual BEV that starts charging at time $k$ and operates at power level $D_{j}$ at a later time $t$ (Qian et al. 2011).

Finally, the probability of a battery charge starting at time instant $k(k \leq t, 1 \leq k \leq 24)$ and charging load operating at power level $D_{j}$ at time instant $t$ can be calculated:

$\Phi\left(D_{j}, t\right)=\sum_{k=1}^{t} \mathrm{~F}(\mathrm{k}) \operatorname{prob}\left(S_{j-(t-k)}\right)$

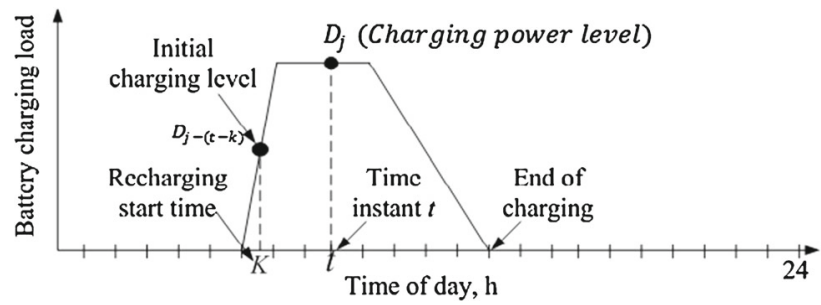

Fig. 1 Schematic of an individual BEV that starts charging at time $k$ and operates at power level $D_{j}$ at a later time $t$ where $\mathrm{F}(\mathrm{k})$ is the probability of charging started at time instant $k(k \leq t)$, which depends on the electricity rate structure in the charging scenario, while $\operatorname{prob}\left(S_{j-(t-k)}\right)$ is the probability of an initial charge being at power level $S_{j-(t-k)}$.

In the proposed spatial-temporal model, the Eq. (5) gives the expression of total batteries charging load in each location:

$\mathrm{P}_{\mathrm{n}}=\sum_{i=1}^{n} \sum_{j=1}^{n_{c}} D_{j} \Phi\left(D_{j}, t\right)$

where $n$ is the total number of BEVs, $n_{c}$ is the total number of half-hourly intervals, and $\mathrm{P}_{\mathrm{n}}$ is the total power demand for the BEV battery chargers. This equation is calculated for hourly recharge in each place that was identified in the spatial distribution.

\section{Modeling of BEV Load Demand Using a Spatial-Temporal Model Under Two Proposed Scenarios}

To minimum data necessary are: network probability for each sub-zone; charging profile of the battery, and maps of the service zone. Most of the data used for this specific study was extracted from Carreno et al. (2011). This information for the proposed model is organized into two databases, spatial and demand, exactly as explained by Melo et al. (2012).

To model the BEV load demand, the city is divided into sub-zones. Each sub-zone has its own load and a network probability. Before starting the modeling, it is necessary to define the expected global load of BEVs for the service zone, i.e., this load expressed as a total penetration of BEVs. In this work, the BEV penetration levels of 10 and $20 \%$ are to be simulated. The BEV battery population is assumed to consist of two different kind of tehcnologies such as $60 \%$ lithium-ion and $40 \%$ of lead-acid.

\subsection{Estimation of the Spatial Distribution of BEVs}

The number of mobile agents coming out of the same subarea is a function of their load density; therefore, sub-areas with high load density may have a greater number of BEVs. It is considered that residents return home to recharge BEVs, these residents could be returning from commercial or industrial zones, it is even possible to consider that some BEVs are used for travel only in residential zones. Thus, all paths of BEVs are considered as short distance trips. At the end of the simulation, the spatial distribution of BEVs will show the sub-zones most likely to have a high agglomeration of BEVs.

At the beginning of the simulation, mobile agent generates a random number $(c)$ between 0 and 1 , which represents the 
SOC if $c=1$, charge full, and if $c=0$ without charge. Then, Eq. (1) is used to calculate the connectedness length; this length determines the number of sub-zones traveled. This procedure characterizes the stochastic nature of its autonomy to move through the city in short distance trips.

A sub-zone is selected from a $3 \times 3$ array. Figure 2 illustrates such an array, in which each position represents the network probability expressed in percentages. In the center of the array is the mobile agent.

To select one sub-zone within the array, some evolutionary elements are used by Glover and Kochenberger (2003). A tournament of two roulette selections is used to specify the winner. The probability of each element on the roulette depends on its network probability. This method is employed to take advantage of controlled randomness and statistical survival-of-the-fittest characteristics. The mobile agent will move to the location of the sub-zone selected. For example, for the previous case, suppose that the winner was the subzone with network probability of $70 \%$ that can be found in the square under of the mobile agent. The mobile agent will move to the position of the sub-zone selected, and the other positions will have their network probabilities assigned to zero, as shown in Fig. 3. This setting allows the representation of BEVs going in one direction and to prevent cycling; thus, different arrivals for BEVs are characterized.

This procedure is randomly controlled, considering the network probability as a control factor. This process is

\begin{tabular}{|l|l|l|l|l|}
\hline $40 \%$ & $60 \%$ & $10 \%$ & $60 \%$ & $10 \%$ \\
\cline { 2 - 4 } $40 \%$ & $40 \%$ & $60 \%$ & $10 \%$ & $10 \%$ \\
\hline $50 \%$ & $50 \%$ & - & $30 \%$ & $30 \%$ \\
\hline $80 \%$ & $80 \%$ & $70 \%$ & $90 \%$ & $90 \%$ \\
\hline $40 \%$ & $60 \%$ & $10 \%$ & $60 \%$ & $10 \%$ \\
\hline
\end{tabular}

Fig. 2 Initial location of the BEV to be simulated

\begin{tabular}{|c|c|c|c|c|}
\hline $40 \%$ & $60 \%$ & $10 \%$ & $60 \%$ & $10 \%$ \\
\cline { 2 - 4 } $40 \%$ & 0 & 0 & 0 & $10 \%$ \\
\hline $50 \%$ & 0 & \multirow{2}{*}{0} & 0 & $30 \%$ \\
\hline $80 \%$ & 0 & $70 \%$ & 0 & $90 \%$ \\
\hline $40 \%$ & $60 \%$ & $10 \%$ & $60 \%$ & $10 \%$ \\
\hline
\end{tabular}

Fig. 3 Initial location of the BEV to be simulated repeated until the mobile agent reaches the boundaries of the service zone or the connectedness length.

\subsection{Modeling of the Hourly Recharge}

Equation (4) uses the probability of charging started at time instant $k$. This likelihood depends on the electricity rate structure, which can be considered in charging scenarios, similar to the one used by Qian et al. (2011).

Two electricity rate structures are considered:

\subsubsection{Flat Rate Scenario}

The BEV owners have no incentives for charging in the offpeak period. In this worst-case scenario BEV owners are expected to begin their recharge cycles at nearly the same time $(6: 00 \mathrm{pm})$; this could be two or three times a week and not daily, and only at their residences. Consequently, a uniform probability density function $\mathrm{F}_{\mathrm{A}(\mathrm{t})}$ of recharging start time is used.

$\mathrm{F}_{\mathrm{A}(\mathrm{t})}=\left\{\begin{array}{ll}1 & \mathrm{t}=18 \\ 0 & \mathrm{t}=\text { any other times }\end{array} 1 \leq \mathrm{t} \leq 24\right.$

\subsubsection{Time of Use Rate Scenario}

In this section, a time-of-use (TOU) electricity rate structure is considered to reflect the existence and possible introduction of new policies to encourage BEV owners to recharge vehicles during the off-peak time. The peak load period is considered to be from 6:00 am to 9:59 pm. A discrete distribution $\mathrm{F}_{\mathrm{B}(\mathrm{t})}$ for BEV off-peak charging is employed to account for the effect of the TOU electricity rate on battery recharging start time.

$\mathrm{F}_{\mathrm{B}(\mathrm{t})}=\left\{\begin{array}{ll}0.33 & \mathrm{t}=22,23,24 \\ 0 & \mathrm{t}=\text { any other times }\end{array} \quad 1 \leq \mathrm{t} \leq 24\right.$

The overall power demand for the BEV battery chargers, at instant $t$, in all scenarios proposed in each place that was identified in the spatial distribution can be calculated based on the distribution of recharge start time, as defined by (6) and (7) above and following the process explained in Sect. 3 .

Figure 4 shows the structure of the overall spatial-temporal model. In this figure, the input and output data from each part explained in Sect. 3 are outlined.

\section{Results}

The methodology was tested using data from a mid-sized city in Brazil with around 200,000 residents.

In this work, the battery population of BEVs is considered to consist of lithium-ion and lead acid. The charging loads 
Fig. 4 Spatial-temporal model

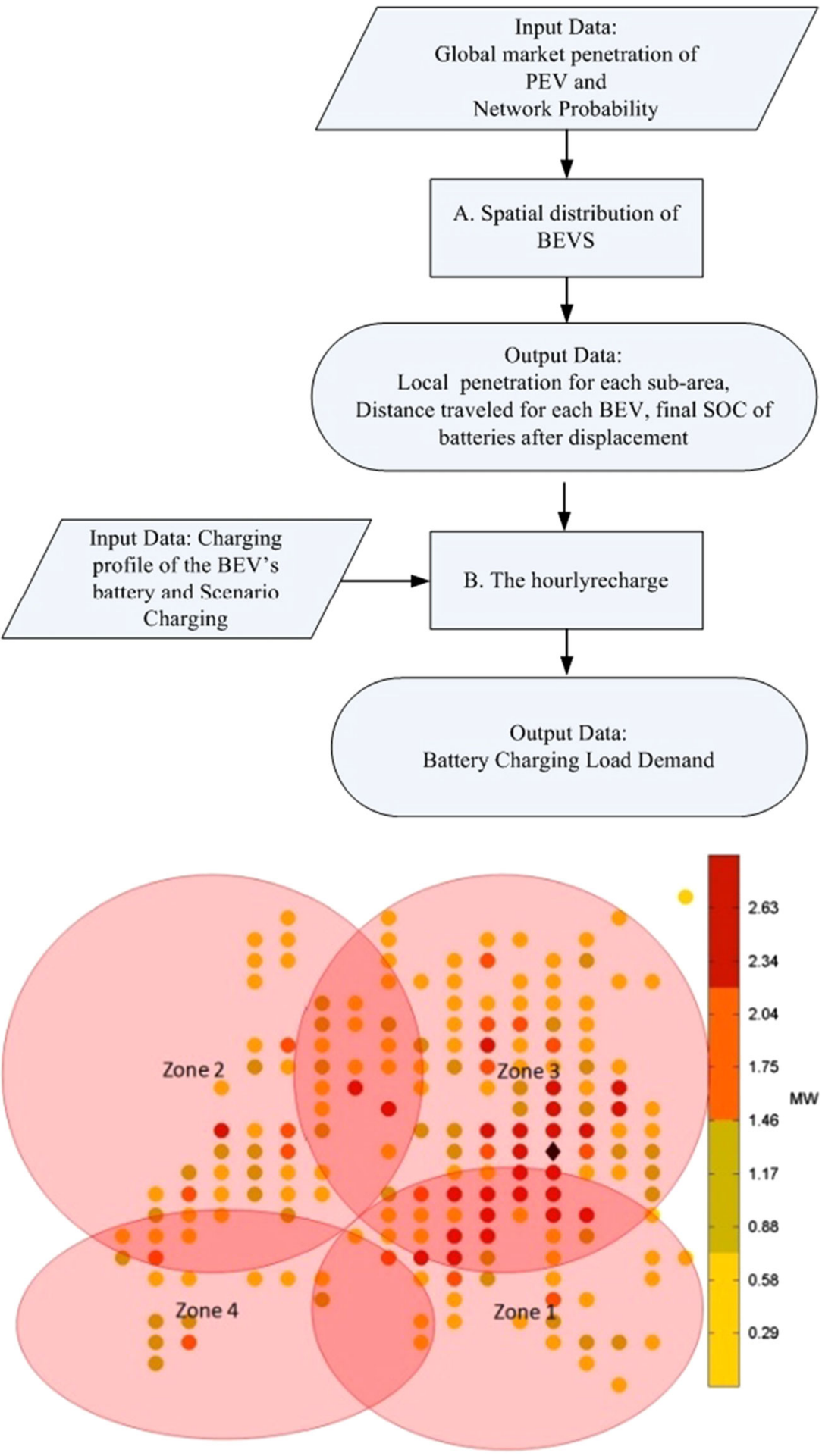

Fig. 5 Real load density for year 2010

for the BEV global penetration levels of 10 and $20 \%$ are simulated. The model can work with different overall levels of penetration depending on the needs of the electric planner.

Figure 5 presents the total load distribution for the year 2010, which is used as the base system. In this figure, the city was divided into four special regions of interest to better analyze the results, which are selected considering the influ- ence area of each of the four existing substations in the base system. The central business district area is identified with a diamond.

Figure 6 present the results of spatial distribution of BEVs using the multi-agent framework explained in Sect. 3.1.2 for a BEV global penetration level of $20 \%$ returning to their residences after a daily commute. Each sub-zone with an 
Fig. 6 Spatial distribution of BEVs to $20 \%$ global penetration level
Fig. 7 Spatial distribution feeders in zone 2
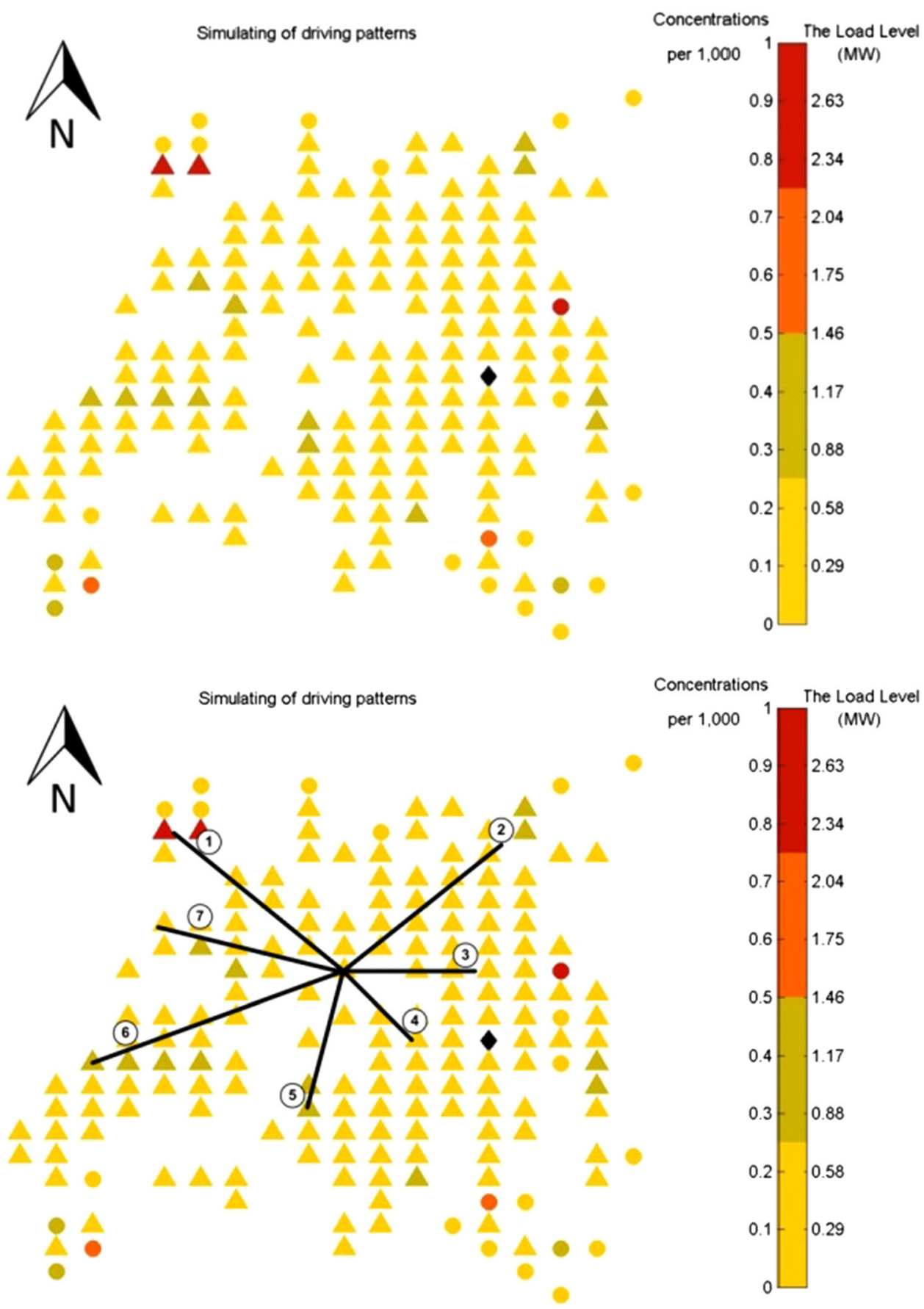

agglomeration of BEVs is identified with a small triangle, and the color scale represents the concentration level. In this figure, each circle represents a regular-sized grid of $0.5 \mathrm{~km}^{2}$. The circular representation is used for visual effect only; the real grid is composed of perfect squares of size $0.71 \mathrm{~km}$. Each part of the grid represents one sub-zone, and the color scale represents the load level. We can observe a significant level of local agglomeration of BEVs in the northern part of the city. Therefore, zones 2 and 3 show a higher agglomeration of BEVs than other areas. Consequently, this distribution is able to show the feeders or distribution transformers where the charging activities will increase the current load demands.

The information obtained from the multi-agent framework could be used to determine charging of BEVs in feeders or distribution transformers, depending on the information available in the database; so the battery charging load obtained by the proposed model can be added to the current load demands in the distribution system. For the application, the BEV charging are presented on all feeders of the zone 2 . Figure 7 shows the spatial distribution feeders in this zone. 


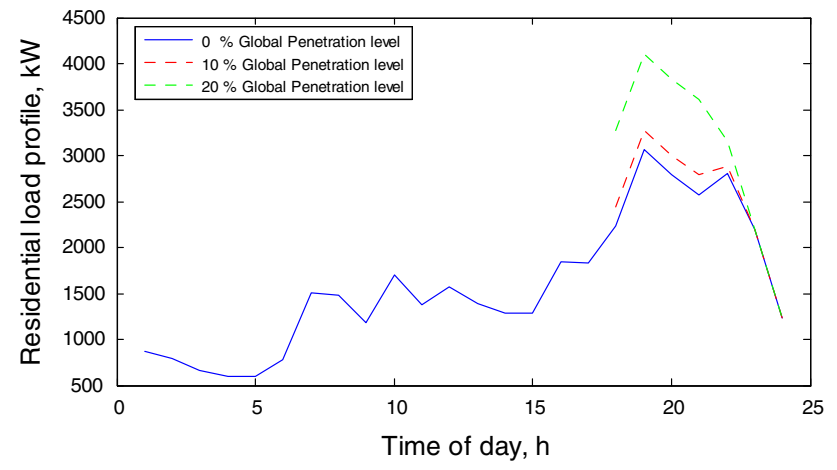

Fig. 8 A - First charging scenario

Table 1 Percentage of increase in daily peak demand on all feeders of Zone 2 for the flat rate scenario

\begin{tabular}{llr}
\hline Feeder & $10(\%)$ & $20(\%)$ \\
\hline Feeder 1 & 7.01 & 34.04 \\
Feeder 2 & 5.03 & 10.31 \\
Feeder 3 & 2.17 & 6.80 \\
Feeder 4 & 1.51 & 4.60 \\
Feeder 5 & 4.27 & 13.68 \\
Feeder 6 & 5.18 & 20.27 \\
Feeder 7 & 1.08 & 11.88 \\
\hline
\end{tabular}

\subsection{Results for Flat Rate Scenario}

Figure 8 shows the results of BEV charging for the first scenario on feeder 1 of Zone 2, using the modeling of the hourly recharge explained in Sect. 4.2. This is a worst-case scenario because all BEVs begin charging at the same time. In this figure, we can observe that peak demand increases up to $34.04 \%$, when the scenario is analyzed with $20 \%$ penetration. The increase in daily peak demand obtained on all feeders of Zone 2 is presented in Table 1. In this table, each column represents the percentage of increase in peak load within the level of global penetration of BEVs. For example, for feeder 6 , a level of $10 \%$ BEV penetration results in an increase upto $5.18 \%$ on peak demand, whereas a level of $20 \% \mathrm{BEV}$ penetration results in an increase upto $20.27 \%$ of the loading during the peak.

\subsection{Results for TOU Charging Scenario}

Figure 9 shows the results of BEV charging for the second scenario on feeder 1 of Zone 2 using the modeling of the hourly recharge explained in Sect. 4.2. In this figure we can observe that a $10 \%$ and a $20 \%$ market global concentration of BEVs in the system did not increase the daily peak demand (around $6 \mathrm{pm}$ ). A $20 \%$ level of BEV penetration would increase demand upto $6.51 \%$ in the new peak demand (around $11 \mathrm{pm}$ ). The increase obtained in the new peak

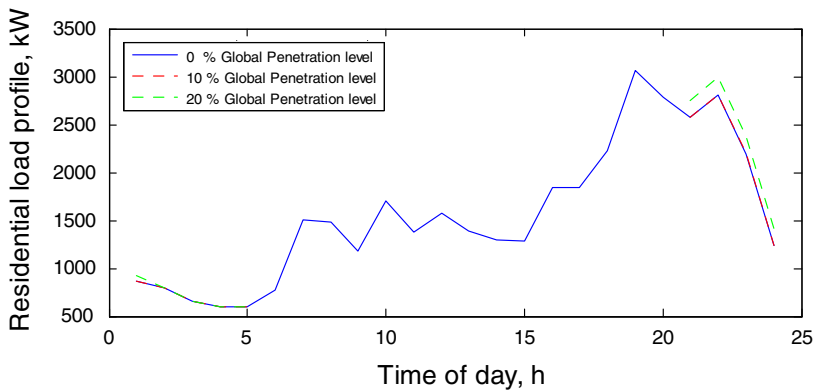

Fig. 9 B - TOU scenario

Table 2 Percentage of increase in daily peak demand on all feeders of Zone 2 for the TOU scenario

\begin{tabular}{lll}
\hline Feeder & $10(\%)$ & $20(\%)$ \\
\hline Feeder 1 & 0.24 & 6.51 \\
Feeder 2 & 0.18 & 1.79 \\
Feeder 3 & 0.10 & 5.60 \\
Feeder 4 & 0.20 & 4.09 \\
Feeder 5 & 0.09 & 5.18 \\
Feeder 6 & 0.35 & 3.33 \\
Feeder 7 & 0.10 & 0.77 \\
\hline
\end{tabular}

demand on all feeders of Zone 2 is presented in Table 2. In this table, each column represents the percentage of increase in system load for the new peak demand within the level of global penetration of BEVs. For example, for feeder 6, the maximum increase will be $3.33 \%$ in the system load with $20 \%$ of BEV penetration during the period between 9:00 pm and midnight. An increase in system load did not exceed the maximum capacity of the feeders, which is about $10 \%$. So, for this scenario a $10 \%$ and a $20 \%$ penetration of BEVs do not cause any increase in peak demand.

As shown in Tables 1 and 2, the most troublesome points are located close to feeder 1 . Higher loadings are observed in feeders with residential loads of Scenario A for a $20 \%$ penetration. The congestion level in Scenario B for all feeders did not exceed its feeder capacity, which is about $10 \%$.

Considering the simplicity of the base system, the computational time is low for any simulation: using a computer with an Intel Core 2 Duo and 3.5 GB of RAM with MATLAB, it takes about 10 and $25 \mathrm{~min}$, for the first and second scenario respectively, to perform all the calculations and visualize the spatial distribution of BEVs and the hourly recharge in each place that was identified in spatial distribution.

\section{Conclusions}

A spatial-temporal approach to estimating the load demand in small scale on a distribution system because of BEV 
charging was presented. The method is able to simulate the driving pattern of BEVs to determine their spatial distribution in a city, and considers the electricity rate structure in two battery charging scenarios. One of the main advantages of this method over others is its ability to determine how the $\mathrm{BEV}$ batteries are charged in small residential areas; at the same time, it simulates the driving patterns to establish the spatial distribution of these vehicles in a city. This distribution enables the identification of small areas with a high penetration of BEVs. These considerations make it possible to model how a particular feeder or a set of transformers could increase the current load demands to accommodate charging of BEVs; other methods simply could not deal with this kind of spatial estimation with only statistical approaches. Therefore, the proposed model contributes to the specialized literature with a novel model that can estimate charging of $\mathrm{BEVs}$ in feeders or transformers.

The spatial simulation considers that the traffic habits are influenced by the behavior of drivers and the electricity rate structure, each with its own philosophy. Thanks to the spatialtemporal nature of traffic habits (Batty 2007), it is possible to simulate the driving patterns of BEVs, mimicking the real behavior of the traffic habits in a real city, and to model the charging of BEV batteries in small areas. This simulation could help electrical utilities in cities without an urban planning area to perform a spatial simulation of BEVs.

For the worst-case scenario with the studied system, a $20 \%$ BEV presence resulted in an increase of $34.04 \%$ in demand during the peak hour in the north of the city, whereas the rest of the city was little affected. The other scenario is less challenging; the increases of electricity consumption had no impact on the daily peak demand.

The results of these kinds of estimations are of great value to utilities in identification of possible overloads in distribution transformers and feeders. This overhead could reduce the useful life of this equipment endangering the operation of the distribution system.

One of the possible drawbacks of this method is the network probabilities, so, future works may focus on improving the aforementioned probabilities.

The spatial-temporal model is compact, with rules that are simple to program. Given a reduced set of data, this system is able to simulate different factors of driving patterns.

Acknowledgments The authors gratefully acknowledge FPTI, CAPES, and CNPq (303817/2012-7, 473679/2013-2) for their economic support.

\section{References}

Batty, M. (2007). Cities and complexity: Understanding cities with cellular automata, agent-based models and fractals. Cambridge: The MIT Press.
Bivand, R. S., Pebesma, E. J., \& Gómez-Rubio, V. (2008). Applied spatial data analysis with $R$ (1ra ed.). New York: Springer.

Broadbent, R. S., \& Hammersley, J. (1957). Percolation processes I: Crystal and mazes. Mathematical Proceedings of The Cambridge Philosophical Society, Julho (pp. 629-641).

Carreno, E. M., Padilha-Feltrin. A. (2009), Spatial electric load forecasting using a local movement approach. International Conference on Intelligent System Applications to Power Systems, IEEE. Curitiba: IEEE, pp. 1-6.

Carreno, E. M., Rocha, R. M., \& Padilha-Feltrin, A. (2011). A cellular automaton approach to spatial electric load forecasting. IEEE Transaction on Power System, 26, 532-540.

Clement-Nyns, K., Haesen, E., \& Driesen, J. (2010). The impact of charging plug-in hybrid electric vehicles on a residential distribution grid. IEEE Transactions on Power Systems, 25(1), 371-380.

Deilami, S., Masoum, A. S., Moses, P. S., \& Masoum, M. A. S. (2011). Real-time coordination of plug-in electric vehicle charging in smart grids to minimize power losses and improve voltage profile. IEEE Transactions Smart Grid, 2(3), 456-467.

Etezadi-Amoli, M., Choma, K., \& Stefani, J. (2010). Rapid-charge electric-vehicle stations. IEEE Transaction on Power Delivery, 25(3), 1883-1887.

Évora-InovCity. (2010)'InovCity". Évora. Janeiro 15, 2010. http:// www.inovcity.pt/pt/Pages/homepage.aspx (accessed Janeiro 12, 2012)

Glover, W., \& Kochenberger, G. A. (2003). Handbook of metaheuristics (1st ed.). Massachusetts: Kluwer Academic Publisher.

Heppenstall, A. J., Crooks, A. T., See, L. M., \& Batty, M. (2012). Agendbased models of geographical systems. Dordrecht: Springer.

KEMA and ISO / RTO Council. (2010) "Assessment of Plug in Electric Vehicle Integratio". ISO/RTO Council. March http://www.isorto.org (accessed 1 Nov 2012)

Khalesian, M. and Delavar, M. R. (2008) "A Multi-Agent based traffic network micro-simulation using spatio-temporal GIS". The international archives of the photogrammetric, remote sensing and spatial information sciences. Beijing: International Society for Photogrammetry and Remote Sensing, pp. 31-36.

Madrid, C., Argueta, J., \& Smith, J. (1999). Performance characterization-1999 Nissan Altra-EV with lithium-ion battery: Technology Report, Southern California EDISON.

Makse, Hernán A, José S Andrade Jr, Michael Batty, Shlomo Havlin, and H Eugene Stanley. (1998) Modeling urban growth patterns with correlated percolation. PHYSICAL REVIEW E 58, no. 6 (December 1998): 7054-7062

Meliopoulos, A. P., J. Meisel, G. Cokkinides, and T. J. Overbye. (2009) Power system level impacts of plug-in hybrid vehicles". Power Systems Engineering Research Center. October. http://www.pserc.org (accessed 15 Jan 2012)

Melo, J. D., E. M. Carreno, and A. Padilha-Feltrin. (2012) A multiagent system with a percolation approach to simulate the driving pattern of plug-in electric vehicles". Transmission and Distribution Conference and Exposition 2012, IEEE. Orlando: IEEE (pp. 1-7).

Mendoza, A., \& Argueta, J. (2000). Performance characterization-GM EV1 Panasonic lead acid battery. Technology Report, Southern California EDISON

Pritchett, A. et al. (2002) Examining air transportation safety issues through agent-based simulation incorporating human performance models". In Proceedings of the IEEE/AIAA 21th Digital Avionics Systems Conference. Irvine (pp. 7A5-1 - 7A5-13), California: IEEE.

Qian, K., Zhou, C., \& Yuan, Y. (2011). Modeling of load demand due to $\mathrm{EV}$ battery charging in distribution systems. IEEE Transactions on Power Systems, 26(2), 802-810.

Rahman, S., \& Shrestha, G. B. (1993). An investigation into the impact of electric vehicle load on the electric utility distribution system. IEEE Transactions on Power Delivery, 8(2), 591-597. 
Roe, C., A. P. Meliopoulos, J. Meisel, and T. Overbye. (2008) Power system level impacts of plug-in hybrid electric vehicles using simulation data. Energy 2030 Conference, IEEE. Atlanta (pp. 1-6), GA: IEEE.

Staats, P. T., Grady, W. M., Arapostathis, A., \& Thallam, R. S. (1997). A procedure for derating a substation transformer in the presence of widespread electric vehicle battery charging. IEEE Transactions on Power Delivery, 12(4), 1562-1568.
Tuttle, D. P., \& Baldick, R. (2012). The evolution of plug-in electric vehicle-grid interactions. IEEE Transactions on Smart Grid, 3(1), 500-505.

Valsera-Naranjo, E., A. Sumper, P. Lloret-Gallego, R. VillafáfilaRobles, and A. Sudria-Andreu. (2009) Electrical vehicles: State of art and issues for their connection to the network. Electrical power quality and utilisation. Lodz (pp. 1-3), Poland: IEEE. 